\title{
SEMI-STABLE AND STABLE CACTI
}

\author{
K. L. MCAVANEY
}

(Received 21 May 1974)

\section{Introduction}

Holton (1973) introduced the following concept. A graph $G$ is semi-stable if there exists a point $v$ in $G$ for which $\Gamma\left(G_{v}\right)=\Gamma(G)_{v}$ : where $\Gamma(G)$ is the automorphism group of $G, G_{v}$ is the graph $G$ with $v$ deleted and $\Gamma(G)_{v}$ is the subgroup of $\Gamma(G)$ that fixes $v$. We say $G$ is semi-stable at $v$. A partial stabilising sequence in $G$ is a sequence $v_{1}, v_{2}, \cdots v_{k}$ of its points such that $\Gamma(G)_{v_{1} v_{2} \cdots v_{i}}=$ $\Gamma\left(G_{v, v_{2} \cdots v_{i}}\right)$ for $i=1,2, \cdots, k$. If there exists a partial stabilising sequence in $G$ for which $k$ equals the number of points of $G$ then $G$ is said to be stable (Holton (1973a)). Most notation and terminology in what follows is explained in Harary (1969).

It is known (Heffernan (1972), Robertson and Zimmer (1972)) that all trees except the paths $P_{n}$ with $n>3$ and the smallest identity tree ( $T_{2}$ in Figure 6) are semi-stable. We showed in McAvaney, Grant and Holton (1974) that the only unicyclic graphs that are not semi-stable are those in Figure 1. In Section 3 we show that these are the only cacti with a cycle that are not semi-stable. (A cactus is a connected graph in which each line lies on at most one cycle).

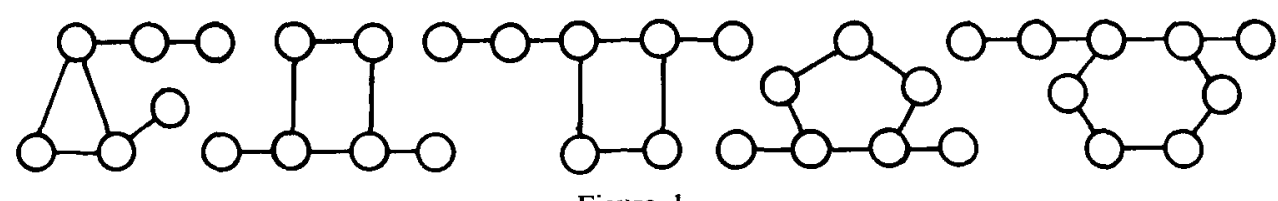

Figure 1.

If a graph is stable then it has a transposition automorphism, Holton and Grant (to appear). The converse is true for trees, Holton (1973b) and unicyclic graphs, McAvaney, Grant and Holton (1974). In Section 4 we show that it is true for all cacti.

Throughout the following sections, we use implicitly a characteristic of semi-stability demonstrated in Holton and Grant (1975): a graph $G$ is 
semi-stable at $v$ if and only if $v$ is an isolated point or the set of points adjacent to $V$ is a union of orbits of $\Gamma\left(G_{v}\right)$. We also use the following terminology. If $v_{1}$, $v_{2}, \cdots, v_{k}$ is a partial stabilising sequence in a graph $G$ and $H=G_{v_{1} v_{2} \cdots v_{k}}$, we say $G$ is reducible to $H$. The distance between a point $v$ in $G$ and a disjoint set $A$ of points in $G$ is the minimum $d(v, a)$ over all points $a$ in $A$. A penultimate point $v$ in $G$ is a point in $G$ such that $G_{v}$ contains just two components one of which is a single point. If the number of points in graph $G$ is less than the number of points in graph $H$, we say $G$ is smaller than $H$. We denote by $\underline{P}_{n}$, a path $P_{n}$ rooted at its endpoint.

\section{Preliminaries}

Before proving our main results we need to establish three lemmas. They require the following ideas. A branch at $a$ point $b$ of a cactus $C$ is a maximal subcactus $B$ of $C$ with two or more points such that just one block of $B$ contains $b$. A branch at $a$ block $D$ of $C$ is a maximal subcactus of $C$ with two or more points and which has just one point $b$ in common with $D$. In both cases $b$ is called the root of the branch.

Lemma 1. A rooted cactus $C$ is semi-stable at a point $c$ which is not a cutpoint or the root.

Proof. Let $b_{1}$ be the root of $C$ and $B_{1}$ a smallest branch at $b_{1}$. Let $B$ denote the block in $B_{1}$ that contains $b_{1}$. Let $b_{2}$ be a cutpoint in $B$ closest to $b_{1}$ and let $B_{2}^{\prime}$ be the branch at $B$ containing $b_{2}$. If $b_{2}^{\prime}$ is another cutpoint in $B$ such that $d\left(b_{1}, b_{2}\right)=d\left(b_{1}, b_{2}^{\prime}\right)$ we assume $B_{2}^{\prime}$ is not larger than the branch at $B$ containing $b_{2}^{\prime}$. If $B_{2}^{\prime}$ is $\underline{P}_{2}$ and $C$ is not semi-stable at its endpoint we redefine $b_{2}$ as a next closest cutpoint (if it exists) to $b_{1}$ and redefine $B_{2}^{\prime}$ accordingly. Let $B_{2}$ denote a smallest branch at $b_{2}$ that does not contain $b_{1}$. Thus, repeating this procedure, we generate a sequence of cutpoints $b_{2}, b_{3}, \cdots, b_{n}$ with associated branches $B_{1}, B_{2}, \cdots, B_{n}$, where $B_{n}$ has just one cutpoint $b_{n}$.

Now every automorphism of $C$ maintains the distance of each point from $b_{1}$. Moreover, a path between $b_{1}$ and a point $a$ of $C$ contains all the cutpoints $w$ such that $b_{1}$ and $a$ lie in different components of $C_{w}$. Hence, from the choice by size of $B_{i}$, if $B_{i}$ is semi-stable at a point $c$ then $C$ is semi-stable at $c$. Therefore $C$ is semi-stable at a point in $B_{n}$ that is adjacent to $b_{n}$, unless $B_{n}$ is $\underline{P}_{2}$ and the block $D$ that contains $b_{n}$ and $b_{n-1}$ is a cycle and contains no other cutpoints. To examine the latter case let $m$ and $l$ denote the number of consecutive points in $D$ between $b_{n}$ and $b_{n-1}$. We may assume $m>l$, for if $m=l$ then $B_{n-1}$, and hence $C$, is semi-stable at the endpoint of $B_{n}$. Then $B_{n-1}$, hence $C$, is semi-stable at $c$ defined as follows: 
if $l=0, m \neq 2$, the bare point adjacent to $b_{n-1}$,

if $l=0, m=2$, the bare point adjacent to $b_{n}$,

if $l=1$, the bare point adjacent to $b_{n-1}$ and $b_{n}$,

if $l>1$, the bare point adjacent to $b_{n-1}$ and farthest from $b_{n}$.

The next lemma requires the following concepts. A cactus $C$ rooted at $b$ and containing at least one copy of a cycle $U$ is called a $U$-pole if (i) all blocks in $C$ are $U$ or $P_{2}$, (ii) all points in the block-cutpoint tree (Harary (1969)) of $C$, $b c(C)$, have degree at most 3 and (iii) if $u$ is a point in $b c(C)$ of degree 3 then $u$ is a copy of $U$ and of the three branches at $u$ one contains $b$, one contains a copy of $U$ and one is $\underline{P}_{2}$. $C$ is called a $U$-pillar if, for all pairs of points in the block-cutpoint tree of $C$ that are copies of $U$, one lies on the path between the other and the block containing $b$.

Lemma 2. A U-pillar is reducible to a $U$-pole.

Proof. Let $C$ be the $U$-pillar and $b_{1}$ its root. By Lemma 1, we reduce to $b_{1}$, in increasing order of size, each branch at $b_{1}$ that does not contain a copy of $U$. Let $D$ be the block that contains $b_{1}$. We define $b_{2}$ and $B_{2}^{\prime}$ as in the proof of Lemma 1. If $B_{2}^{\prime}$ does not contain $U$ we reduce it to $\underline{P}_{2}$. If $C$ is now semi-stable at the endpoint of $B_{2}^{\prime}$, we remove it. Otherwise we redefine $b_{2}$ as the next closest cutpoint to $b_{1}$ and redefine $B_{2}^{\prime}$ accordingly. Then we reduce $B_{2}^{\prime}$ to $b_{2}$ and repeat this procedure until all branches (except possibly for a single $\underline{P}_{2}$ ) that do not contain $U$ are removed from $D$. Finally, let $b_{2}$ denote the root of the branch (if it exists) at $D$ that contains $U$. Then $b_{2}$ is fixed in $C$ and we repeat the above procedure on all branches at $b_{2}$ except the branch containing $b_{1}$. In this way we generate a sequence of cutpoints $b_{2}, b_{3}, \cdots, b_{n}$ where $b_{n}$ lies in a copy of $U$.

If the block containing $b_{i}$ and $b_{i+1}$ is a cycle which is not $U$ and has $\underline{P}_{2}$ as a branch, let $b_{i}^{\prime}$ denote the root of that $\underline{P}_{2}$. Then, for each $i$ in turn for which $b_{i}^{\prime}$ is defined, we remove $b_{i}^{\prime}$ followed by the isolated point and reduce the resulting path branches at $b_{i}$ and $b_{i+1}$ to their roots. Similarly, if the cycle containing $b_{i}$ and $b_{i+1}$ is not $U$ and has no branch $\underline{P}_{2}$, we remove a point adjacent to $b_{i}$ and reduce the resulting path branch at $b_{i+1}$ to its root. The resulting cactus is a $U$-pole.

Lemma 3. A $U$-pole is semi-stable at a non-cutpoint $c$ in the copy of $U$ farthest from the root.

Proof. Define $c$ as in the proof of Lemma 1.

\section{Semi-stable Cacti}

Our aim in this section is to show that the graphs in Figure 1 are the only cacti with a cycle that are not semi-stable. We shall use the following notation. Let $C$ denote a cactus with at least one cycle. For a cycle $R$ in $C$, let $n(C, R)$ 
denote the number of copies of $R$ in $C$. Let $m(C)$ be the minimum $n(C, R)$ over all cycles $R$ in $C$. Finally, let $U$ denote the smallest cycle in $C$ for which $n(C, U)=m(C)$.

We first establish

THEOREM 1. If $m(C)=1$ then $C$ is semi-stable unless it is one of the cacti in Figure 1. Moreover $C$ is semi-stable at a point which is a penultimate point or non-cutpoint.

Proof. We assume $m(C)=1$ and that $C$ is not semi-stable. If there is only one branch $B$ at $U$ then its root $b$ is fixed in $C$ and hence, by Lemma $1, B$ is $\underline{P}_{2}$. But then $C$ is semi-stable at $b$. Hence there are at least two branches at $U$.

We shall call a point in $U$ of degree 2 a bare point. Let $t$ denote the maximum number of consecutive bare points in $U$. Then $t>0$; otherwise a smallest branch at $U$ is reducible to its root, by Lemma 1. Let $S$ denote the collection of branches at $U$ whose roots are adjacent to a string of $t$ consecutive bare points. Let $B_{1}$ denote a smallest branch in $S$ and $b_{1}$ its root. Let $B_{2}$ denote a smallest branch in $S$ whose root $b_{2}$ is adjacent to the same string of $t$ consecutive bare points as is the root of a branch in $S$ that is isomorphic to $B_{1}$. We may assume $b_{2}$ is adjacent to the same string of $t$ consecutive bare points as $b_{1}$. Noting that the set of roots of the branches in $S$ is a union of orbits of $\Gamma(C)$, Lemma 1 implies that $B_{1}$ is $\underline{P}_{2}$.

Let $B_{3}$ denote the branch at $U$ whose root $b_{3}$ is the closest to $b_{1}$. Let $r=d\left(b_{1}, b_{3}\right)-1$ (the number of bare points between $b_{1}$ and $b_{3}$ ). Note that $r<t$, for if $r=t$ then $C$ is semi-stable at the endpoint $e$ of $B_{1}$. It follows from the definition of $t$ that the removal of $e$ introduces the "reflection" automorphism $g$ that maps $b_{2}$ into $b_{3}$. Hence $B_{2}$ and $B_{3}$ are isomorphic if not the same branch (see Figure 2).

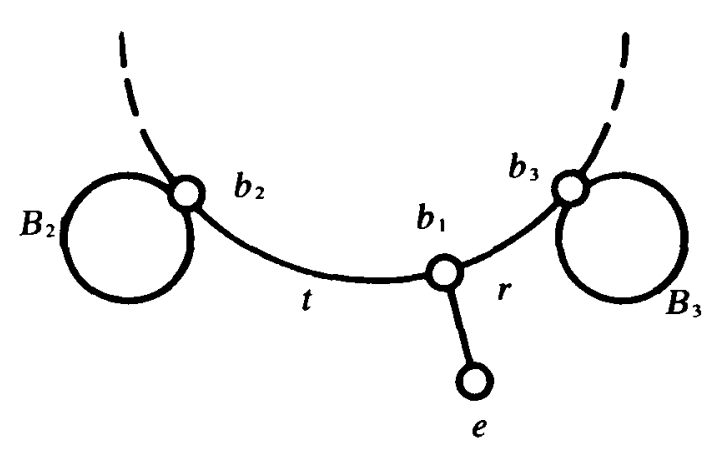

Figure 2. 
It follows from Lemma 1 and the definition of $B_{2}$ that $B_{2}$ has at most 3 points, otherwise $C$ is semi-stable at a point in $B_{2}$. Thus $B_{2}$ is $\underline{P}_{2}, \underline{P}_{3}$ or one of the branches in Figure 3.
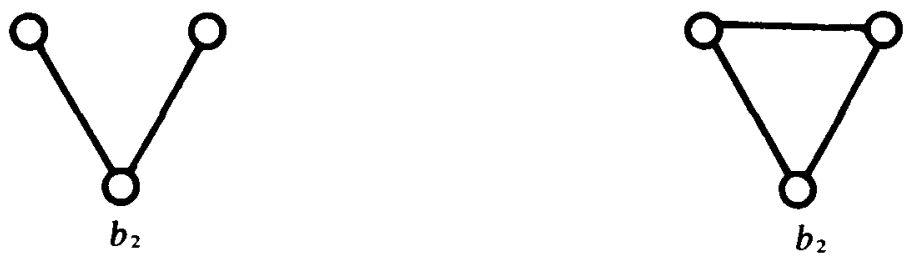

Figure 3.

If $b_{2} \neq b_{3}$ and $B_{2}$ is not $\underline{P}_{2}$, then the removal of one of its non-cutpoints introduces the "reflection" automorphism $f$ that maps $b_{1}$ into $b_{2}$. Define inductively the points $b_{4}, b_{5}, \cdots$ on $U$ as follows: $b_{2 i}=f\left(b_{2 i-1}\right)$ and $b_{2 i+1}=$ $g\left(b_{2 i}\right)$ for $i=1,2,3, \cdots$. Then the branch at $U$ with root $b_{j}$ is isomorphic to $B_{2}$ for $j \neq 1$, and (excluding $b_{1}$ ) all other points on $U$ are bare. But then $C$ is semi-stable at a non-cutpoint in $B_{3}$. Hence $B_{2}$ is $\underline{P}_{2}$ or $b_{2}=b_{3}$. Thus, if $b_{2}=b_{3}, C$ falls intc one of the three cases indicated in Figure 4 which we now examine in turn. (By the definition of $U, B_{2}$ is not isomorphic to the second branch in Figure 3.)
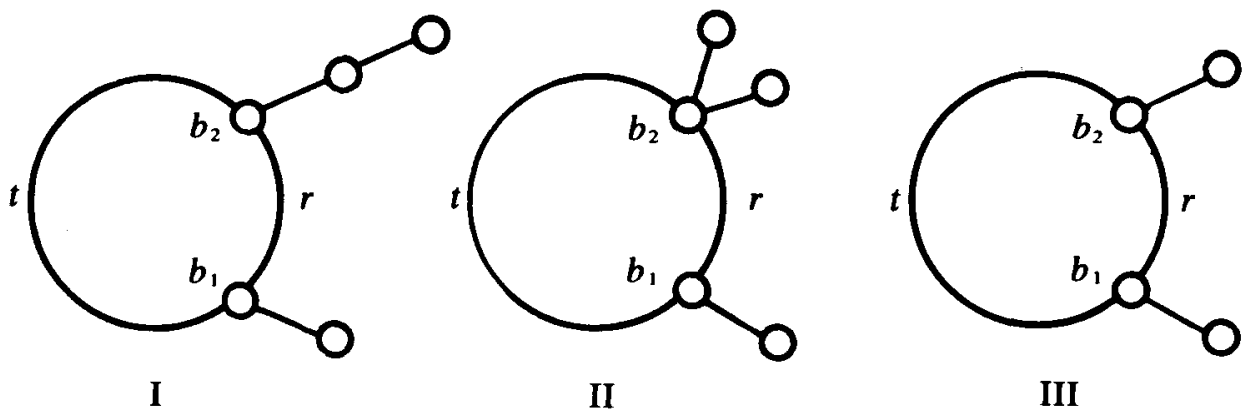

Figure 4.

Case I:

If $r=0$ and $t=1, C$ is the first graph in Figure 1.

If $r=0$ and $t=2, C$ is the third graph in Figure 1 .

If $r=0$ and $t=3, C$ is semi-stable at the bare point adjacent to $b_{2}$.

If $r=0$ and $t=4, C$ is the fifth graph in Figure 1 .

If $r=0$ and $t>4, C$ is semi-stable at the bare point adjacent to $b_{2}$.

If $r=1$ and $t=2, C$ is semi-stable at the bare point adjacent to $b_{\text {, }}$ but not $b_{2}$. 
If $r=1$ and $t>2, C$ is semi-stable at $b_{1}$.

If $r=2, \quad C$ is semi-stable at the bare point adjacent to $b_{1}$ that is closer to $b_{2}$.

If $r>2, \quad C$ is semi-stable at $b_{1}$.

Case II:

If $r=0$ and $t=1, C$ is semi-stable at the bare point.

If $r=0$ and $t>1, C$ is semi-stable at $b_{1}$.

If $r=1, \quad C$ is semi-stable at the bare point adjacent to $b_{1}$ and $b_{2}$.

If $r>1, \quad C$ is semi-stable at $b_{1}$.

Case III:

If $r=0$ and $t=1, C$ is semi-stable at the bare point.

If $r=0$ and $t=2, C$ is the second graph in Figure 1 .

If $r=0$ and $t=3, C$ is the fourth graph in Figure 1.

If $r=0$ and $t>3, C$ is semi-stable at the bare point adjacent to $b_{1}$.

If $r=1, C$ is semi-stable at the bare point adjacent to $b_{1}$ and $b_{2}$.

If $r>1, C$ is semi-stable at $b_{1}$.

If $b_{2} \neq b_{3}$ and $B_{2}$ is $\underline{P}_{2}$ then the removal of its endpoint introduces the "reflection" automorphism $h$ that maps $b_{1}$ into $a_{4}$, the point in $U$ that is closest to $b_{2}$ and which is not a bare point. Thus we define inductively the points $a_{4}, a_{5}$, $a_{6}, \cdots$ in $U$ as follows: $g\left(b_{2}\right)=b_{3}, h\left(b_{1}\right)=a_{4}, g\left(a_{4}\right)=a_{5}, h\left(b_{3}\right)=a_{6}$, etc. Then the branches at $U$ with roots $b_{1}, b_{2}, b_{3}, a_{i}(j \geqq 4)$ are $\underline{P}_{2}$ and all other points in $U$ are bare (see Figure 5). If $s=d\left(b_{2}, a_{4}\right)-1$ then $s<t$ and we may assume that $s \leqq r$. Thus:

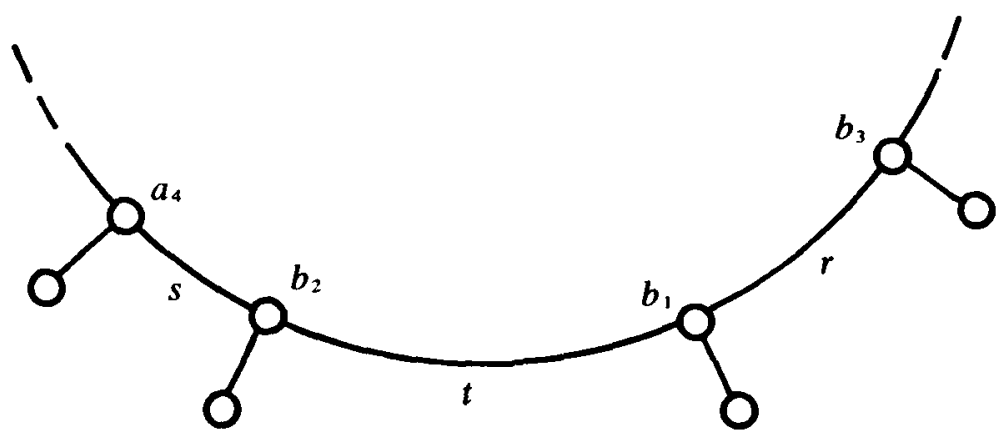

IV

Figure 5 . 
Case IV:

If $r=0$ and $t=1, C$ is semi-stable at the bare point.

If $r=0$ and $t>1, C$ is semi-stable at $b_{3}$.

If $r=1, \quad C$ is semi-stable at the bare point adjacent to $b_{1}$ and $b_{3}$.

If $r>1, \quad C$ is semi-stable at $b_{1}$.

This completes the proof of Theorem 1.

Our second main result is

THEOREM 2. If $m(C)>1$ then $C$ is reducible to a cactus $C^{\prime}$ for which $m\left(C^{\prime}\right)=m(C)-1$.

ProOf. The rationale of this proof is to remove a sufficient number of branches of $C$ in order to allow us to remove a point of some copy of $U$. The resulting cactus suffices for $C^{\prime}$. A variety of cases present themselves according to the distribution in $C$ of its copies of $U$.

Let $b c(C)$ denote the block-cutpoint tree of $C$. Let $N$ be the set of points in $b c(C)$ which are either copies of $U$ or points of degree 3 or more and at which there exists 3 or more distinct branches each containing a copy of $U$. Let $T(C, U)$ denote the tree whose points are the points in $N$ and in which two points $a, b$ are adjacent if and only if the path in $b c(C)$ joining $a$ to $b$ does not contain a point in $N$. Finally, let $E$ be the centre of $T(C, U)$.

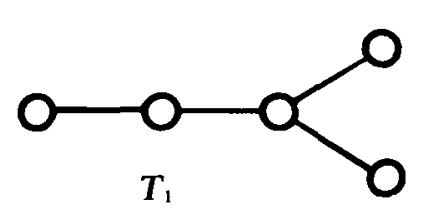

$T_{1}$

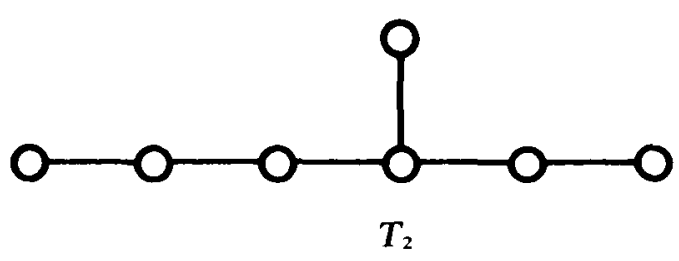

Figure 6 .

We now assume for the moment that $T(C, U)$ is not a path $P_{n}(n \geqq 2)$ or one of the trees $T_{1}$ or $T_{2}$ in Figure 6. Heffernan (1972) has shown that all trees $T$ except $P_{n}(n>2), T_{1}$ and $T_{2}$ are semi-stable at an endpoint $u$. His argument is constructive and ensures that $u$ lies in a smallest branch at each point $v$ on the path in $T$ from $u$ to the closer centre point in $T$. We tighten this condition for $T(C, U)$ by choosing from the smallest branches at $v$ a branch that corresponds to a smallest branch at $v$ in $C$. We note that $u$ is a copy of $U$.

Let $w$ be the point in $T(C, U)$ that is adjacent to $u$. If $w$ is not in $E$, let $a$ denote the point of $C$ in $w$ that is closest to $E$ in $C$. If $w$ is in $E$ and $T(C, U)$ is 
bicentral, let $a$ denote the point of $C$ in $w$ that is closest to $E \backslash\{w\}$ in $C$. In both cases let $b$ denote the point of $C$ in $w$ that is closest to $u$ in $C$. If $w$ is acycle in $C$, let $b^{\prime}$ denote the point of $C$ in $w$ for which $d(a, b)=d\left(a, b^{\prime}\right)$. Let $B\left(B^{\prime}\right)$ denote the maximal subcactus of $C$ that contains $b\left(b^{\prime}\right)$ but not the rest of the branch at $b$ that contains $E$. If $B^{\prime}$ contains one copy of $U$, then by the definition of $u$, we may assume $B^{\prime}$ is not smaller than $B$. Thus we may regard $b$ as fixed in $C$ and use Lemma 2 to reduce $B$ to a $U$-pole at $b$. If $B^{\prime}$ contains no copy of $U$, we use Lemma 1 to reduce $B^{\prime}$ to its root. By Lemma 3, $B$ is semi-stable at a non-cutpoint $c$ in $u$. Hence, from the definition of $u, C$ is semi-stable at $c$.

If $w$ is in $E$ and $T(C, U)$ is unicentral, let $b$ denote the point of $C$ in $w$ that is closest to $u$. By Lemma 2, we may assume the branch $B$ at $w$ that contains $u$ is a $U$-pole. (Also, if $w$ is a point in $C$, we use Lemma 1 to reduce to $b$ the branches at $w$, in increasing order of size, that do not contain a copy of $U$.) Let $c$ be a point in $u$ at which $B$ is semi-stable. Then two cases present themselves.

Case I: $w$ is not fixed in $C_{c}$. Then there are just three branches in $T(C, U)$ at $w$. Let $v$ and $v^{\prime}$ be the other two points in $T(C, U)$ adjacent to $w$. Let $A\left(A^{\prime}\right)$ be the branch in $C$ at $w$ that contains $v\left(v^{\prime}\right)$ and let $a\left(a^{\prime}\right)$ be its root. Let $e\left(e^{\prime}\right)$ denote the point in $v\left(v^{\prime}\right)$ that is closest to $a\left(a^{\prime}\right)$. Let $w$ map into $w^{\prime}$ in $C_{\mathrm{c}}$. We assume that $w^{\prime}$ is in $A^{\prime}$. Thus $d\left(a^{\prime}, e^{\prime}\right)>d(a, e)$ and $a^{\prime}$ is therefore fixed in $C$. Then by the methods of Lemma 2, we reduce $A^{\prime}$ so that all points between $a^{\prime}$ and $e^{\prime}$ have degree 2 and no branches remain at $e^{\prime}$ that do not contain a copy of $U$. Note that, while reducing $A^{\prime}$ in this way, $d\left(a^{\prime}, e^{\prime}\right)$ is non-decreasing. Thus we may assume the $w^{\prime}$, and hence $w$, is a point in $C$. But then $w(=b)$ can not map into $w^{\prime}$ as $B_{c}$ is a branch that does not contain a copy of $U$ and is not smaller than $\underline{P}_{2}$ and no such branch exists at a point between $a^{\prime}$ and $e^{\prime}$. Hence $C$ is now semi-stable at $c$.

Case II: $w$ is fixed in $C_{c}$. If $w$ is a point in $C$ we reduce, in increasing order of size, all the branches at $w$ that do not contain $U$. Then $C$ is semi-stable at $c$ or Case I applies.

If $w$ is a cycle in $C$, we use the methods of Theorem 1. Define a bare point as a point in $w$ of degree 2 or a root of a branch at $w$ that contains $U$. Then, as in the proof of Theorem 1, several cases present themselves. Note that there are at least three branches at $w$ containing $U$.

Case II. 1: There is no branch at $w$ not containing $U$. Then $C$ is semi-stable at $c$.

Case II. 2: There is only one branch $A$ at $w$ not containing $U$ and it is $\underline{P}_{2}$. If $C$ is not semi-stable at $c$ then $B_{c}$ is $\underline{P}_{2}$ and $B$ is $Q$, the second branch in Figure 3. If $B$ is the only branch at $w$ isomorphic to $Q$ and $C$ is not semi-stable at the endpoint of $A$, then a "reflection" argument similar to that in the proof of Theorem 1 guarantees at least three isomorphic branches at $w$ containing $U$. 
Let $B^{\prime}$ be one of these branches with its root closest to the root of $A$. Then, noting that the root of $A$ is fixed in $C$, we use Lemma 1 to reduce $B^{\prime}$ to $Q$ or until a point in a copy of $U$ is removed. If there are at least two branches at $w$ isomorphic to $Q$ and $C$ is not semi-stable at a point with degree 2 in any of them, then another "reflection" argument shows that their roots together with the root of $A$ are distributed uniformly in $w$ a constant distance apart. Hence we may remove the endpoint of $A$ and then Case II.1 applies.

Case II.3: There are only two branches $A$ and $A^{\prime}$ at $w$ not containing $U ; A$ is $\underline{P}_{2}$ and $A^{\prime}$ is $\underline{P}_{2}, \underline{P}_{3}$ or one of the branches in Figure 3. If $C$ is not semi-stable at $c$ then $B_{c}$ maps into $A$ or $A^{\prime}$. If the former, we may remove the endpoint of $A$ and then Case II.2 applies. If the latter, then because $B$ is a $U$-pole, $A^{\prime}$ is either $\underline{P}_{2}$ or $\underline{P}_{3}$. Hence we may remove respectively the endpoint or point of degree 2 in $A^{\prime}$ and then Case II.2 applies.

Case II.4: There are three or more branches at $w$ not containing $U$. These are distributed about $w$ as in Case IV of Theorem 1 . If $C$ is not semi-stable at $c$ then $B$ is $C_{3}$ (rooted at one point) and $b$ is one of the $t$ consecutive bare points at distance $r+1$ from $b_{2}$ or distance $s+1$ from $b_{1}$. But then $C$ is semi-stable at the endpoint of $B_{1}$ or $B_{2}$ respectively, giving a contradiction.

To complete the proof of Theorem 2 we now investigate the cases where $T(C, U)$ is $P_{n}(n \geqq 2), T_{1}$ or $T_{2}$ (Figure 6).

If $T(C, U)$ is $P_{2}$, let $u$ and $u^{\prime}$ be the copies of $U$. Let $b^{\prime}(b)$ be the point of $C$ in $u\left(u^{\prime}\right)$ closest to $u^{\prime}(u)$. Let $B\left(B^{\prime}\right)$ be the maximal subcactus of $C$ that contains $b\left(b^{\prime}\right)$ but no other point in the branch $A\left(A^{\prime}\right)$ at $b\left(b^{\prime}\right)$ that contains $u^{\prime}(u)$. We assume $B$ is not larger than $B^{\prime}$ and use Lemma 2 to reduce $B$ to a $U$-pole. Then, by the methods of Lemma $2, A$ is reducible to $u^{\prime}$ with at most two other points, that is, at most one more point than $A^{\prime}$. Let $c$ be a point in $u$ at which $B$ is semi-stable. If $C$ is not semi-stable at $c$ then $B_{c}$ maps into a branch at $u^{\prime}$. Then $B_{c}$ is $\underline{P}_{2}, \underline{P}_{3}$ or one of the branches in Figure 3 . The cacti that satisfy these constraints consist of just two copies of either $C_{3}$ or $C_{4}$ together with at most three other points. It can be shown exhaustively that all such cacti are semi-stable at some point in $u$ or $u^{\prime}$.

If $T(C, U)$ is $P_{n}$ with $n>2$ let $u$ and $u^{\prime}$ be the endpoints of $T(C, U)$. Let $v\left(v^{\prime}\right)$ be the point in $T(C, U)$ adjacent to $u\left(u^{\prime}\right)$. Let $b\left(b^{\prime}\right)$ be the point of $C$ in $v\left(v^{\prime}\right)$ closest to $u^{\prime}(u)$. Let $B\left(B^{\prime}\right)$ be the maximal subcactus of $C$ containing $b\left(b^{\prime}\right)$ but no other point in the branch at $b\left(b^{\prime}\right)$ that contains $u^{\prime}(u)$. We assume $B$ is not greater than $B^{\prime}$ and use Lemma 2 to reduce $B$ to a $U$-pole. Let $a\left(a^{\prime}\right)$ be the point of $C$ in $u^{\prime}(u)$ closest to $u\left(u^{\prime}\right)$ and let $A\left(A^{\prime}\right)$ be the maximal subcactus of $C$ that contains $a\left(a^{\prime}\right)$ but no other point of the branch at $a\left(a^{\prime}\right)$ that contains $u\left(u^{\prime}\right)$. Then, by the methods of Lemma $2, A$ is reducible to $u^{\prime}$ with at most two other points, that is, at most one more point than $A^{\prime}$. Let $c$ be a point in $u$ at which $B$ is semi-stable. If $C$ is not semi-stable at $c$ then $B_{c}$ maps 
into $A$. Hence $B_{c}$ consists of $v$ with a branch that is $\underline{P}_{2}, \underline{P}_{3}$ or one of the branches in Figure 3. Hence $U$ is $C_{3}$ or $C_{4}$, and it can be shown exhaustively that all cacti satisfying these constraints are semi-stable at some point in $u, u^{\prime}$ or $v$.

If $T(C, U)$ is $T_{1}$ (Figure 6) let $w$ be the point in $T(C, U)$ of degree 3 and $v$ the point of degree 2. Let $a$ denote the point of $C$ in $w$ closest to $v$. Let $A$ denote the branch of $C$ at $w$ that contains $v$. Since $a$ is fixed in $C$, we may use Lemma 2 to reduce $A$ to a $U$-pole. Let $B$ and $B^{\prime}$ be the branches at $w$, with roots $b$ and $b^{\prime}$ respectively, that contain one copy of $U$. Assuming $B$ is not larger than $B^{\prime}$ we reduce $B$ to a $U$-pole. Let $e$ be the point of $C$ in $w$ for which $d(a, b)=d(a, e)$. In increasing order of size, the branches at $e$ that do not contain $U$ are reduced to $e$ using Lemma 1 . Let $c$ be a point in $u$, the copy of $U$ in $B$, at which $B$ is semi-stable. Suppose $C$ is not semi-stable at $c$. If $w$ is a point in $C$, then $w=a=b$ and maps, in $C_{c}$, into a point $w^{\prime}$ between $v$ and the end copy of $U$ in $A$. But $B_{c}$ is not smaller than $\underline{P}_{2}$ and no branch exists at $w^{\prime}$ that does not contain $U$. Herice $w$ is a cycle, and $w$ maps into $v$ in $C_{c}$. Then there is a branch $\underline{P}_{2}$ at $v, B_{c}$ is $\underline{P}_{2}$ and therefore $U$ is $C_{3}$. Hence, in reducing $A$ to a $U$-pole, we can reduce $\underline{P}_{2}$ at $v$ to its root. This contradiction ensures $C$ is semi-stable at $c$.

If $T(C, U)$ is $T_{2}$ (Figure 6) let $w$ be the point in $T(C, U)$ of degree 3. Let $A(B)$ be the branch at $w$, with root $a(b)$, that contains one (two) copy(ies) of $U$. Using Lemma 2 we may reduce $A$ and $B$ to $U$-poles. Let $c$ be a point in the end copy of $U$ in $B$ at which $B$ is semi-stable. If $C$ is not semi-stable at $c$ then $B_{c}$ maps into $A$, there is a branch $\underline{P}_{z}$ at the end copy of $U$ in $A$ and $U$ in $C_{3}$. Then $C$ is semi-stable at the point of degree 2 in the penultimate copy of $U$ in $B$.

This concludes the proof of Theorem 2.

As a corollary to Theorem 2 we have

THEOREM 3. All cacti $C$ with at least one cycle are semi-stable except those in Figure 1. Also, $C$ is semi-stable at a point which is a penultimate point or non-cutpoint.

Proof. If $m(C)=1$ then the result follows immediately from Theorem 1 . In the case where $m(C)>1$ the proof of Theorem 2 tacitly secures the required point.

\section{Stable Cacti}

Our aim now is to show that a cactus with a transposition automorphism is stable. We first characterise these cacti. To do this, we define a certain class of subcacti. A cactus $C$ is said to contain a transfig at a point $b$ if there is (i) a branch at $b$ isomorphic to $C_{3}$ (rooted at one point), or (ii) a branch in which the 
block $D$ containing $b$ is $C_{4}$ and in which the two points in $D$ adjacent to $b$ have degree 2 , or (iii) two or more branches at $b$ isomorphic to $P_{2}$, or (iv) any combination of (i), (ii) and (iii).

We note that, in this section, the cacti include trees.

Theorem 4. A cactus $C$ with at least three points has a transposition automorphism if and only if it contains a transfig.

Proof. If $C$ contains a transfig and $a$ and $a^{\prime}$ denote its points of degree 2 (in types (i) and (ii)) or its endpoints (in type (iii)), then clearly the transposition $\left(a a^{\prime}\right)$ is an automorphism of $C$.

Conversely, suppose $g=\left(a a^{\prime}\right)$ is a transposition automorphism of $C$. Then, because $C$ is connected and it has at least three points, there is another point $u$ of $C$ adjacent to $a$ or $a^{\prime}$. Suppose $u \sim a$. Then $g(u) \sim g(a)$, that is $u \sim a^{\prime}$. If $a \sim a^{\prime}$, then $u$ is unique, otherwise the line $\left(a, a^{\prime}\right)$ lies on more than one cycle in $C$. If $a \not a^{\prime}$, then there is at most one other point $u^{\prime}$ of $C$ for which $u^{\prime} \sim a$ and $u^{\prime} \sim a^{\prime}$, otherwise the line $(u, a)$ lies on more than one cycle in $C$. Either way $u$ is a root of a transfig.

Using this characterisation we can now prove

THEOREм 5. A cactus containing just one transfig is stable.

Proof. Let $C$ denote such a cactus and $b$ the root of the transfig. Let $A$ denote the maximal subcactus of $C$ that contains $b$ but not the rest of the transfig. If $A_{b}$ is empty and $C$ is $C_{3}$ or $C_{4}$ then clearly $C$ is stable. If $A_{b}$ is empty and $C$ is neither $C_{3}$ nor $C_{4}$ then $b$ is fixed and, by Lemma $1, C$ is reducible to one of the cacti in Figure 7. These cacti are stable; we delete the points in the indicated order.
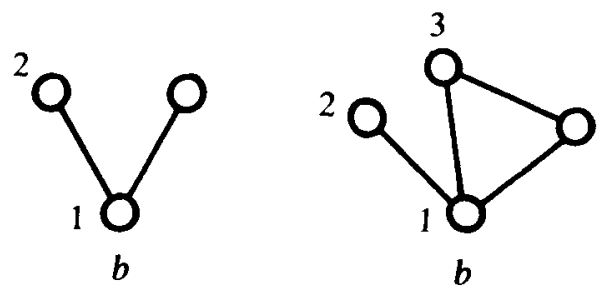

Figure 7

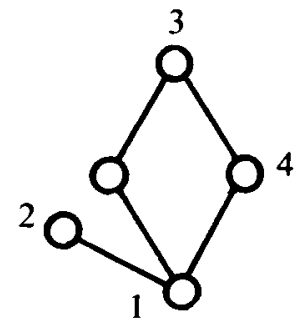

b

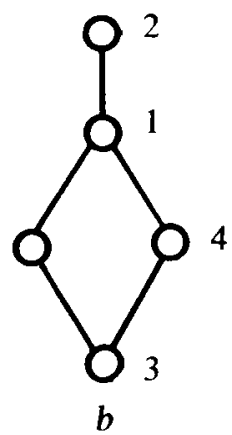

$b$

Suppose now that $A_{b}$ is not empty. Since $A$ contains no transfig it is reducible, by Lemma 1 , to $\underline{P}_{3}$ or the rooted cactus $A^{\prime}$ (Figure 8 ). In the latter case we then remove the cutpoint $u$ followed by the isolated point, thus leaving $P_{3}$. We assume in the case where the transfig is of type (ii), that $A$ is not larger than the other branch at $C_{4}$. 
If the resulting cactus is $B$ in Figure 8 , we continue the stabilising sequence as indicated. Otherwise we remove the point of degree 2 in $A$ followed by the isolated point. Then, as before, $C$ is reducible to one of the cacti in Figure 7 and hence stable.

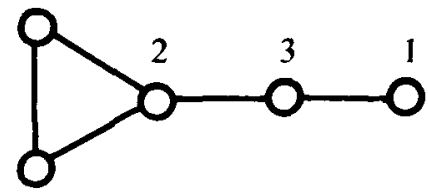

B

Figure 8.

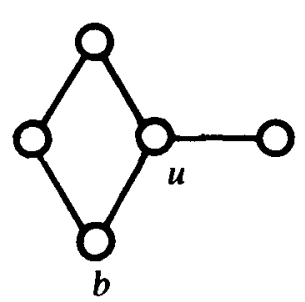

$\mathbf{A}^{\prime}$

Combining Theorem 5 and Theorem 3 we have

THeOREM 6. A cactus $C$ is stable if and only if it has a transposition automorphism.

Proof. We may assume from Theorem 4 and Theorem 5 that $C$ has at least two transfigs. Then, by Theorem 3 and the analogous result (Heffernan (1972)) for trees, $C$ is semi-stable at a non-cutpoint or penultimate point $v$. If $C_{v}$ contains just one transfig (after removing any isolated point), the result follows from Theorem 5 . Otherwise we continue reducing $C$ until only one transfig remains.

\section{References}

F. Harary (1969), Graph Theory (Addison-Wesley, 1969).

P. Heffernan (1972), Trees (Masters Dissertation, University of Canterbury, New Zealand).

D. A. Holton (1973), 'Two applications of semi-stable graphs', Discrete Math. 4, 151-158.

D. A. Holton (1973a), 'A report on stable graphs', J. Austral. Math. Soc. 15, 163-171.

D. A. Holton (1973b), 'Stable trees', J. Austral. Math. Soc. 15, 476-481.

D. A. Holton and D. D. Grant (1975), 'Regular graphs and stability', J. Austral. Math. Soc. 20, 377-384.

D. A. Holton and D. D. Grant, (to appear), 'Products of graphs and stability', J. Combinatorial Theory.

K. L. McAvaney, D. D. Grant and D. A. Holton (1974), 'Stable and semi-stable unicyclic graphs', Discrete Math. 9, 277-288.

N. Robertson and J. A. Zimmer (1972), 'Automorphisms of subgraphs obtained by deleting a pendant vertex', J. Combinatorial Theory Ser. B. 12, 169-173.

\section{Mathematics Department}

Gordon Institute of Technology

Geelong, Vic. 3220

Australia. 